\title{
Detection of Illicit Drugs using Surface Enhanced Raman Spectroscopy
}

\author{
Richa Goel, Vimarsh Awasthi and Satish Kumar Dubey* \\ Centre for Sensors, Instrumentation and Cyber Physical System Engineering (SeNSE), Indian \\ Institute of Technology Delhi, India
}

*Corresponding author: Satish Kumar Dubey, Assistant Professor, Centre for SeNSE, IIT Delhi, Delhi, India, Tel: +91-11-26596752; Email: satishdubey@sense.iitd.ac.in

\section{Review Article \\ Volume 6 Issue 1}

Received Date: December 14, 2020

Published Date: January 18, 2021

DOI: $10.23880 / \mathrm{ijfsc}-16000220$

\section{Abstract}

Rapid identification of drugs of abuse in different types of forensic evidences, including bulk street drugs as well as traces found in drinks, on fibers/clothing, in fingerprints, on fingernails, on bank notes, in body fluids etc., is of critical importance. Current forensic methods involved in identification and quantification of illicit drugs are destructive and slow, making it difficult for them to be used for rapid on-site analysis. Surface enhanced Raman Spectroscopy (SERS) offers distinct advantages to various law-enforcement agencies, working in the security field, as it provides rapid, sensitive, accurate and non-destructive technique for the identification and quantification of a variety of drug types. This review summarizes techniques for detection of contraband drugs, difficulties associated with them and discusses the future direction of research in this field

Keywords: SERS; Drugs; SORS; Plasmonics

Abbreviations: SERS: Surface Enhanced Raman Spectroscopy; FTIR: Fourier Transform Infrared; DUID: Driving under the Influence of Drugs; TERS: Tip Enhanced Raman Scattering; CARS: Coherent Anti-Stokes Raman Scattering; SRS: Stimulated Raman Scattering; HRS: Hyper Raman Scattering; SORS: Spatially offset Raman Spectroscopy.

\section{Introduction}

The intrinsic features of Raman spectroscopy along with the instrumental advancements have triggered the application of this spectroscopic tool in many fields and are transforming it from a technique accessible to a small number of specialized users, to a more widely available analytical technique. It has become a relatively common tool used by a number of agencies to identify powders suspected of being biological agents [1,2], explosives [3,4], or drugs [5], simply by exposing the sample to a monochromatic laser light source. The photons interacting with the sample molecule can undergo Rayleigh scattering (occurring at the same wavelength as the incident light) or Raman scattering (occurring at slightly higher (Stokes) or slightly lower (anti-Stokes) wavelengths than the incident light source). The shifts and intensities of these wavelengths (Stokes or anti-stokes) in Raman scattering are characteristic of each molecule, indicating the types of bonding present in the target molecule thereby enabling identification of the chemicals.

It is found that only one in every $10^{6}-10^{8}$ photons will Raman scatter, which makes Raman signals very weak in nature and vulnerable to being masked by fluorescence, thus its capability to detect trace quantities of drugs on surfaces that are essentially invisible, is incapacitated. However, Raman scattered signals can be significantly enhanced by the interaction of chemicals with the plasmon field generated by lasers at the surface of gold or silver nanoparticles to enhance the Raman signal intensity by as much as six- 


\section{International Journal of Forensic Sciences}

eight orders of magnitude [6,7]. This phenomenon is called as surface-enhanced Raman spectroscopy (SERS). SERS was discovered accidently while pyridine was studied on roughened silver electrode [8]. SERS spectroscopy is one of the most powerful analytical techniques which can be used for the identification of molecular species, with the potential to reach single-molecule detection under ambient conditions [9]. The advantage of SERS over other ultrasensitive techniques, such as mass spectrometry, is that it provides an excellent opportunity to acquire the spectra directly from aqueous solution. SERS is a phenomenon in which the Raman signals of molecules are enormously enhanced when they are placed in the vicinity of SERS-active nanostructures. In due course SERS will become a versatile analytical tool in various fields such as surface sciences, material sciences, life sciences, environmental sciences, forensic sciences and food sciences and eventually may help in developing commercial instrument that is widely used in medicine, energy, agriculture, pollution, security and criminal investigation.

The application of SERS to illicit drug detection began at the turn of the century and included detection/identification of cocaine, barbiturates, and amphetamines [5,10-12]. The widespread use of illicit drugs is enormously responsible for crime and violence in society, is dangerous to public health, is a threat to the safety and welfare of mankind and can endanger the security and sovereignty of countries $[13,14]$. It is therefore a major concern amongst law enforcing agencies all across the globe.

Currentforensicmethodsfordetectingillicitdrugsinclude, colour tests, microcrystal tests, high performance liquid chromatography $[15,16]$, ultra violet spectrophotometry $[17,18]$, coupled gas chromatography mass spectroscopy [19], Fourier transform infrared (FTIR) spectroscopy [20] and powder X-Ray diffraction. Though, chromatographic techniques have high sensitivity and molecular specificity, but they involve pre-processing techniques such as sample preparation, separation of materials, besides, the reagents used may be toxic and hazardous to the operator and to the environment. These techniques are thus time consuming and destructive, making it difficult to take them out of the lab and carry out on-site analysis. The requirement of rapid, sensitive, accurate, non-destructive and on-site analysis for the trace of illegal drugs to ensure public safety has inspired the scientific community to explore new instruments and methodologies in this direction.

SERS by virtue of its high sensitivity and unique spectroscopic fingerprint is capable of bridging this gap. It can be developed as simple, rapid and cost-effective tools for the sensitive detection and identification of contraband drugs in seized samples, including hidden compounds in legal materials such as beverages and clothes, detection of trace samples found in drinks, in fingerprints, on envelopes, on bank notes etc. Quantitative SERS can also be employed to determine the actual drug concentrations in street cocaine, crack rocks etc. and to identify possible adulterants in these samples for forensic toxicology and criminalistics. Here we review the different technologies available, their applications vis-à-vis drug detection and their drawbacks.

\section{Drug Menace}

Driving under the influence of drugs (DUID) is turning out to be a big menace to the society, Studies have revealed that the number of deaths resulting of car accident with DUID has surpassed the death number with drunken driving. In $2015,43 \%$ of fatally injured motorists were found to be driving under the influence of drugs, while $37 \%$ of motorists who died had consumed alcohol [21]. DUID poses a serious threat to the public and property safety. So far, there has been no specific methods for detecting the presence of on-site drug testing for suspected drug drivers. Generally, tests based on blood and urine samples are conducted, but such tests with blood sample needs trained medical staff to operate, which might otherwise impose a risk of infection. Likewise, urine test though can be used to detect drugs and metabolites, however, it involves privacy issues. Both the methods require sample collection and laboratory analysis, and consume a long inspection cycle. Law enforcement agencies are in dire need of rapid screening technique for on-site and roadside identification of DUID.

Measurement of illicit drugs on paper currency is of interest for evidentiary purposes in legal cases involving the drug trade. Handling or abuse of a drug leads to contamination of fingers. Analysis of fingernails and fingerprints, coupled with detection/characterization of the substance embedded in it could possibly help in establishing a link between a suspect and the substance of abuse and can be used in breaking the smuggling racquet.

In the fight against smuggling, analysis of cocaine in alcoholic drinks in sealed bottles is becoming a top priority amongst various government agencies. The ability of smugglers to conceal large quantities of drugs in a single bottle, which could be hidden in a genuine consignment of drink bottles makes detection almost impossible without opening each individual bottle. Displaced Raman spectroscopy, a variant of spatially offset Raman Spectroscopy, is not only quick and non-destructive technique, but it is also immune to fluorescence emissions of bottle. It can be used to detect cocaine dissolved in rum contained in transparent bottles without the need for opening the seal of the bottle.

Detection of Gamma-hydroxy-butyric acid (GHB), a central nervous system depressant, is another major concern 


\section{International Journal of Forensic Sciences}

amongst law-enforcing agencies in their fight against drugfacilitated sexual assaults. GHB, usually administered with alcoholic drinks as its sedative affect tends to get accentuated in its presence. It is used for spiking the drinks. Benchtop and portable Raman spectrometers has been used for analyzing this drug in a variety of containers such as glass/plastic vials/plastic bags etc [22].

\section{Saliva as Drug Specimen}

Using saliva as a biological specimen for testing of drugs offers a very viable option as it offers several advantages such as: it is non-invasive, sample collection is easier than for urine or blood samples, does not involve infringement of privacy and it has less interference from impurities. However, saliva is plagued with few drawbacks, such as (i) drug concentration is relatively lower in saliva as compared to blood or urine thus, the need to develop highly sensitive detection technique, (ii) the collection method of saliva will have a significant effect on the outcome of the test (iii) there has been a steady inflow of synthetic drugs, which requires instrument to detect multiple drugs simultaneously (iv) for rapid screening of people on road, we need faster detection technique $[23,24]$. SERS is capable of addressing these issues with equanimity.

Saliva samples from 44 narcotic users and 52 non-users were examined using SERS and the samples from the users showed a characteristic band at $1030 \mathrm{~cm}^{-1}$ that was not observed in the non-users [25]. Thus, it is possible to rapidly identify users and nonusers with this technique. Analysis of saliva by SERS can detect usage of heroin, methamphetamine and methadone [26,27]. SERS is capable of providing improved and reliable on-site drug-detection technique. Recent developments in portable Raman spectrometers and advancements in data analysis offer exciting opportunities for new applications of Raman spectroscopy in the identification and quantification of drugs of abuse, including investigations conducted immediately at the scene of a crime. A recent study has used two commercially available Raman spectrometers in order to detect street samples of cocaine, ecstasy, and amphetamine in situ in an international airport arrivals area [28]. Spectra were obtained and identified within 30s or less without requiring the sample to be removed from its container. This reduces the potential for contamination and also has the advantage that the operator does not come into contact with an unknown substance that could possibly be harmful.

\section{Current SERS Techniques Involved in Drug Detection}

Nowadays, scientists are working on several aspects of SERS, for example, fundamental aspects related to the electromagnetic or the chemical enhancement mechanisms, single molecule detection, structural-property investigations with the objective to explore how the structure of SERS substrate influences its optical response, Tip enhanced Raman scattering (TERS) [29], and ultrafast SERS studies of molecular dynamics at the interface with metallic surfaces. The plasmonic amplification of the optical response has also been exploited to enhance coherent anti-Stokes Raman scattering (CARS) [30], stimulated Raman scattering (SRS) [31], hyper Raman scattering (HRS) [32], fluorescence, and infrared absorption. With the proliferation of commercial availability of portable or handheld spectrometers the possibility of carrying out on-site analysis is becoming increasingly feasible [33-35]. Besides, there is a surge in the so-called hyphenated instruments, that combine Raman with other analytical techniques, one such instrument is a combination of Fourier transform infrared and Raman instrument [36], such instruments allow to perform characterization of the sample as per our requirements. The manufacturers allow the flexibility to modify the features of these instruments (weight, sensitivity, excitation wavelength, price, etc.) as per our requirements for specific application.

Another important field is the development of substrates with optimal characteristics for SERS; fabrication strategies have been reviewed in several papers which include, wet chemical protocols, the assembly of nanoparticles on different types of surfaces, and the fabrication of ordered arrays of nanoparticles [37-39].

A number of papers have proposed new SERS-active media for the detection of drug traces. SERS data for analysis of two different forms of cocaine in solution using colloidal silver has been reported. It was found that SERS can easily distinguish the two forms of cocaine even in the presence of adulterants such as lidocaine or benzocaine, common cutting agents for cocaine [40-42]. The SERS spectra obtained for lower concentrations of cocaine solution indicated that the technique may be useful for forensics.

SERS substrate based on Paper dipstick or swab with inkjet-printed plasmonic substrate, utilizing lateral- flow concentration has been demonstrated with detection limits as low as $95 \mathrm{fg}$ of R6G, $413 \mathrm{pg}$ of malathion, $15 \mathrm{ng}$ of cocaine, and $9 \mathrm{ng}$ of heroin [43]. These paper-SERS devices are easy to fabricate, have sensitivity comparable to conventional SERS substrates and traditional microfluidic devices, are inexpensive and may find immediate applications for remote locations or on-site trace chemical analysis in liquid samples or on surfaces, including the detection of illegal drugs by law enforcement agencies [44].

SERS detection of heroin, codeine and cocaine samples achieved detection limit of $0.2 \mu \mathrm{g} / \mathrm{mm}^{2}$ [45]. The 


\section{International Journal of Forensic Sciences}

detection limit of 19 and $17 \mathrm{ppm}$ for amphetamine and methamphetamine using a coupling of the amines with 2-mercaptonicotinic acid prior to the surface derivatization has been reported [46]. By measuring SERS directly in drug solutions the spectral differences of morphine, codeine and hydrocodone has been demonstrated [47]. Commercially available SERS substrates has been used to determine the limit of detection, limit of identification and dynamic range for common narcotic drug analytes, including cocaine, amphetamine and morphine [48]. These papers and many more [49-54] are clear indicators of the emergence of SERS in narco-analysis.

Spatially offset Raman Spectroscopy (SORS) has emerged as a promising technology for detection and quantification of analytes. It has successfully examined cocaine dissolved in alcoholic drink (rum) inside transparent glass bottle with detection limit to be of the order of $9 \mathrm{~g}$ of pure cocaine per $0.7 \mathrm{l}$ of rum in less than $1 \mathrm{~s}$ [55]. Researchers have used SORS technique in analysis of drugs from various sources such as: multilayered postal package, identification of a medicine inside its plastic blister pack; analysis of an envelope containing unknown substance; and identification of a drug dissolved in a clear solvent, contained in a nontransparent plastic bottle, noninvasive quantification of ternary drug mixtures inside an opaque plastic container $[56,57]$.

The need of rapid and accurate on-site analysis can be fulfilled by portable/hand-help Raman spectrometer. Detection of cocaine $\mathrm{HCl}$, MET and amphetamine sulfate with unknown constituents in less than a minute has been demonstrated using portable Raman spectrometer in real airport environment by comparing the measured spectra with the available data [27]. The need of transportable Raman spectrometer at borders have encouraged researchers to detect and seize drugs at borders under real conditions and parameters [58]. Researchers are exploring ways to use multi wavelengths for excitation instead of sticking to $785 \mathrm{~nm}$ source, (considered as an industrial standard') as it shows strong fluorescence. A new dispersive Raman spectrometer using excitation wavelength as 1064 $\mathrm{nm}$ demonstrated that Raman bands of heroin and cannabis could be clearly identified unlike in the case of $785 \mathrm{~nm}$ wavelength demonstrating the major advantage of the 1064 $\mathrm{nm}$ excitation for the analysis of these types of drugs despite the longer integration time required to obtain the spectra [59-61].

Integration of data processing and analysis with Raman instrumentation to enhance the overall efficiency is soon becoming inevitable. Several search algorithms based on conventional Euclidean distance searching, spectral angle mapping and correlation algorithms were tested on spectral library [62] to identifying illegal substances deposited in fingerprints based on the infrared absorption features that can easily be applied to Raman features. It has also been demonstrated how preprocessing techniques such as truncating, Savitzky-Golay smoothing, normalization, differentiating, mean centering etc. would help in resolving the spectral differences between various samples with almost $100 \%$ accuracy [63].

\section{Conclusion}

Applications of SERS as a non-destructive, rapid and sensitive method for detection and classification of drugs has made significant progress in the past decade. This has helped preserve the forensic evidences, recovered at crime scenes and maintain the chain of custody. The technique is capable of detection and quantification of trace samples. Rapid progress in instrumentation will witness SERS moving out of the lab environment and deployed in the field for forensic and strategic applications. Integration of artificial intelligence with SERS active device will ensure identification and classification of even concealed drugs of abuse and aid the government agencies to lead their offensive at all places including airports, borders, post offices and other public places. SERS is capable of identifying all sorts of illicit drugs in concealed containers, in the presence of adulterants, does not generate any chemical waste, does not require any preprocessing or sample preparation, hence eliminating any possibility of being hazardous to the environment. By implementing SERS for the detection of drugs of abuse, we are sure to win our fight against drug-trafficking and save our future generations from the clasp of such menace.

\section{Conflicts of Interests}

Authors declare no conflict of interest.

\section{Funding}

Authors gratefully acknowledge the financial assistance from Department of Science and Technology, Delhi, Government of India for the Project No. RP03496.

\section{References}

1. Liu B, Zhou P, Liu X, Sun X, Li H, et al. (2013) Detection of Pesticides in Fruits by Surface-Enhanced Raman Spectroscopy Coupled with Gold Nanostructures. Food and Bioprocess Technology 6(3): 710-718.

2. Baniukevic J, Hakki Boyaci I, Goktug Bozkurt A, Tamer U, Ramanavicius A, et al. (2013) Magnetic gold nanoparticles in SERS-based sandwich immunoassay for antigen detection by well oriented antibodies. Biosensors and Bioelectronics 43(1): 281-288. 


\section{International Journal of Forensic Sciences}

3. Dasary SSR, Singh AK, Senapati D, Yu H, Ray PC (2009) Gold nanoparticle based label-free SERS probe for ultrasensitive and selective detection of trinitrotoluene. Journal of the American Chemical Society 131(38): 13806-13812.

4. Goel R, Awasthi V, Rai P, Dubey SK (2020) Numerical Design of Photonic Crystal-Based Nanostructured Substrate for Efficient Surface-Enhanced Raman Scattering. Plasmonics, pp: 1-8.

5. F Aljanabi (2016) Detection of cocaine using gravure printed silver nanoparticle-based SERS substrate. IEEE SENSORS.

6. Nie S, Emory SR (1997) Probing single molecules and single nanoparticles by surface-enhanced Raman scattering. Science 275(5303): 1102-1106.

7. Kneipp K (1997) Single molecule detection using surface-enhanced raman scattering (SERS). Physical Review Letters 78(9): 1667-1670.

8. Jeanmaire DL, Van Duyne RP (1977) Surface Raman spectro-electrochemistry: part I. heterocyclic, aromatic, and aliphatic amines adsorbed on the anodized silver electrode. J Electroanal Chem Interfacial Electrochem 84(1): 1-20.

9. Kneipp J, Kneipp H, Kneipp K (2008) SERS-a singlemolecule and nanoscale tool for bioanalytics. Chemical Society Reviews 37(5): 1052-1060.

10. Piotr Piotrowski, Jolanta Bukowska (2015) 2-Mercaptoethanesulfonate (MES) anion-functionalized silver nanoparticles as an efficient SERS-based sensor of metal cations. Sensors and Actuators B: Chemical 221: 700-707.

11. Nutt DJ, King LA, Phillips LD (2010) Drug harms in the UK: A multicriteria decision analysis. Lancet 376(9752): 1558-1565.

12. Sordo L, Indave BI, Barrio G, Degenhardt L, de la Fuente L, et al. (2014) Cocaine use and risk of stroke: a systematic review. Drug Alcohol Depen 142: 1-13.

13. Davis P, Niaz K, Pietschmann T, Shelton J, Vella A (2014) World Drug Report, United Nations Office on Drugs Crime. UN.

14. Chaiken JM, Chaiken MR (1990) Drugs and predatory crime. Crime Justice 13: 203-239.

15. Dwight Stoll R, Xiaoping Li, Xiaoli Wang, Peter Carr W, Sarah Porter EG, et al. (2006) Fast, comprehensive twodimensional liquid chromatography. J Chromatogr A
1168(1-2): 3-43.

16. Stoll DR, Paek C, Carr PW (2006) Fast Gradient Elution Reversed-Phase Liquid Chromatography with DiodeArray Detection as a High-throughput Screening Method for Drugs of Abuse II. Data Analysis. J Chromatogr A 1137(2): 163-172.

17. Roux C, Bull S, Goulding J, Lennard CJ (2000) Tracing the source of illicit drugs through plastic packaging--a database. Forensic Sci 45(1): 99-114.

18. Furnari C, Ottaviano V, Rosati F, Tondi V (1998) Identification of 3,4-methylenedioxyamphetamine analogs encountered in clandestine tablets. Forensic Sci Int 92(1): 49-58.

19. Toennes SW, Kauert GF, Steinmeyer S, Moeller MR (2005) Driving under the influence of drugs -- evaluation of analytical data of drugs in oral fluid, serum and urine, and correlation with impairment symptoms. Forensic Sci Int 152(2-3): 149-155.

20. Willer U, Saraji M, Khorsandi A, Geiser P, Schade W (2006) Near-and mid-infrared laser monitoring of industrial processes, environment and security applications. Opt Lasers Eng 44(7): 699-710.

21. Hedlund J (2017) Drug-inpaired Driving. Washington. DC.

22. Brewster VL, Edwards HGM, Hargreaves MD, Munshi T (2009) Identification of the date-rape drug GHB and its precursor GBL by Raman spectroscopy. Drug Test Anal 1(1): 25-31.

23. Tang MHY, Ching CK, Poon S, Chan SSS, Ng WY, et al. (2018) Evaluation of three rapid oral fluid test devices on the screening of multiple drugs of abuse including ketamine. Forensic Sci Int 286: 113-120.

24. Quintela 0, Crouch DJ, Andrenyak DM (2006) Recovery of drugs of abuse from the immunalysis Quantisal oral fluid collection device. J Anal Toxicol 30(8): 614-616.

25. Anyu C, Lin H, Zi Jian C, Yi J, Dian Q, et al. (2009) Detecting Narcotic Usage Using Surface-Enhanced Raman Spectroscopy on Saliva Samples. IFMBE Proc 25(7): 7174.

26. Plouffe BD, Murthy SK (2017) Fluorescence-based lateral flow assays for rapid oral fluid roadside detection of cannabis use. Electrophoresis 38(3-4): 501-506.

27. Verstraete AG (2005) Oral fluid testing for driving under the influence of drugs: history, recent progress and remaining challenges. Forensic Sci Int 150(2-3): 143- 


\section{International Journal of Forensic Sciences}

150.

28. Hargreaves MD, Page K, Munshi T, Tomsett R, Lynch G, et al. (2008) Analysis of seized drugs using portable Raman spectroscopy in an airport environment-a proof of principle study. J Raman Spectrosc 39(7): 873-880.

29. Kumar N, Weckhuysen BM, Wain AJ (2019) Nanoscale chemical imaging using tip-enhanced Raman spectroscopy. Nat Protoc 14: 1169-1193.

30. Zumbusch A, Holtom GR, Xie XS (1999) ThreeDimensional Vibrational Imaging by Coherent AntiStokes Raman Scattering. Physical Review Letters 82(20): 4142-4145.

31. Freudiger CW, Min W, Saar BG, Lu S, Holtom GR, et al. (2008) Label-free biomedical imaging with high sensitivity by stimulated raman scattering microscopy. Science 322(5909): 1857-1861.

32. Denisov VN, Mavrin BN, Podobedov VB (1987) HyperRaman scattering by vibrational excitations in crystals, glasses and liquids. In Physics Reports 151(1): 1-92.

33. Carron K, Cox R (2010) Qualitative Analysis and the Answer Box: A Perspective on Portable Raman Spectroscopy. Anal Chem 82(9): 3419-3425.

34. Pilot R (2018) SERS detection of food contaminants by means of portable Raman instruments. J. Raman Spectrosc 49(6): 954-981.

35. Zheng J, Pang S, Labuza TP, He L (2014) Evaluation of surface-enhanced Raman scattering detection using a handheld and a bench-top Raman spectrometer: A comparative study. Talanta 129: 79-85.

36. Ellis DI, Muhamadali H, Haughey SA, Elliott CT, Goodacre R (2015) Point-and-shoot: Rapid quantitative detection methods for on-site food fraud analysis-Moving out of the laboratory and into the food supply chain. Anal Methods 7(22): 9401-9414.

37. Stockman MI, Shalaev VM, Moskovits M, Botet R, George TF (1992) Enhanced Raman scattering by fractal clusters: Scale-invariant theory. Physical Review B 46(5): 2821-2830.

38. Itoh T, Ozaki Y, Yoshikawa H, Ihama T, Masuhara H (2006) Hyper-Rayleigh scattering and hyper-Raman scattering of dye-adsorbed silver nanoparticles induced by a focused continuous-wave near-infrared laser. Applied Physics Letters 88(8): 084102.

39. Kneipp H, Kneipp K, Seifert F (1993) Surface-enhanced hyper-Raman scattering (SEHRS) and surface-enhanced
Raman scattering (SERS) by means of mode-locked Ti:sapphire laser excitation. Chemical Physics Letters 212(3-4): 374-378.

40. Carter JC, Brewer WE, Angel SM (2000) Raman Spectroscopy for the in Situ Identification of Cocaine and Selected Adulterants. Appl Spectrosc 54(12): 1876.

41. SM Angel, Carter JC, Stratis DN, Marquardt BJ, Brewer WE (1990) Some new uses for filtered fiber-optic Raman probes: in situ drug identification and in situ and remote Raman imaging. J Raman Spectrosc 30(9): 795-805.

42. Dana K, Shende C, Huang H, Farquharson S (2015) Rapid Analysis of Cocaine in Saliva by Surface-Enhanced Raman Spectroscopy. Journal of analytical \& bioanalytical techniques 6(6): 1-5.

43. Ameku WA, De Araujo WR, Rangel CJ, Ando RA, Paixao TRLC (2019) Gold Nanoparticle Paper-Based DualDetection Device for Forensics Applications. ACS Applied Nano Materials 2(9): 5460-5468.

44. Yu WW, White IM (2013) Inkjet-printed paper-based SERS dipsticks and swabs for trace chemical detection. Analyst 138(4): 1020-1025.

45. Horvath E, Mink J, Kristof J (1997) Surface-enhanced Raman spectroscopy as a technique for drug analysis. Mikrochim Acta 14: 745-746.

46. Sulk RA, Corcoran RC, Carron KT (1999) SurfaceEnhanced Raman Scattering Detection of Amphetamine and Methamphetamine by Modification with 2-Mercaptonicotinic Acid. Appl Spectrosc 53(8): 954959.

47. Rana V, Canamares MV, Kubic T, Leona M, Lombardi JR (2011) Surface-enhanced Raman Spectroscopy for Trace Identification of Controlled Substances: Morphine, Codeine, and Hydrocodone. J Forensic Sci 56(1): 200207.

48. Massarini E, Wasterby P, Landstrom L, Lejon C, Beck 0 , et al. (2015) Methodologies for assessment of limit of detection and limit of identification using surfaceenhanced Raman spectroscopy. Sensor Actuat B Chem 207: 437-446.

49. Sagmuller B, Schwarze B, Brehm G, Trachta G, Schneider $S$ (2003) Identification of illicit drugs by a combination of liquid chromatography and surface-enhanced Raman scattering spectroscopy. J Mol Struct 661: 279.

50. Faulds K, Smith WE, Graham D, Lacey RJ (2002) Assessment of silver and gold substrates for the detection of amphetamine sulfate by surface enhanced 


\section{International Journal of Forensic Sciences}

Raman scattering (SERS). Analyst 127(2): 282-286.

51. Ackermann KR, Henkel T, Popp J (2007) Quantitative Online Detection of Low-Concentrated Drugs via a SERS Microfluidic System. Chem Phys Chem 8(18): 26652670.

52. Chen J, Jiang J, Gao X, Liu G, Shen G, et al. (2008) A New Aptameric Biosensor for Cocaine Based on Surfaceenhanced Raman Scattering Spectroscopy Chem Eur J 14(27): 8374-8382.

53. Farquharson S, Shende C, Sengupta A, Huang H, Inscore F (2011) Rapid detection and identification of overdose drugs in saliva by surface-enhanced Raman scattering using fused gold colloids. Pharmaceutics 3(3): 425-439.

54. Fox JD, Waverka KN, Verbeck GF (2012) Gold-plating of Mylar lift films to capitalize on surface enhanced Raman spectroscopy for chemical extraction of drug residues. Forensic Sci Int 216(1-3): 141-145.

55. Eliasson C, Macleod NA, Matousek P (2008) Noninvasive detection of cocaine dissolved in beverages using displaced Raman spectroscopy Anal. Chim Acta 607(1): 50-53.

56. Olds WJ, Jaatinen E, Fredericks P, Cletus B, Panayiotou H, et al. (2011) Spatially offset Raman spectroscopy (SORS) for the analysis and detection of packaged pharmaceuticals and concealed drugs Forensic Sci Int 212(1-3): 69-77.

57. Olds WJ, Sundarajoo S, Selby M, Cletus B, Fredericks PM, et al. (2012) Noninvasive, Quantitative Analysis of Drug Mixtures in Containers Using Spatially Offset Raman Spectroscopy (SORS) and Multivariate Statistical Analysis. Appl Spectrosc 66(5): 530-537.

58. Weyermann C, Mimoune Y, Anglada F, Massonnet G, Esseiva P, et al. (2011) Applications of a transportable Raman spectrometer for the in-situ detection of controlled substances at border controls. Forensic Sci Int 209(1-3): 21-28.

59. Hargreaves MD, Burnett AD, Munshi T, Cunningham JE, Linfield EH, et al. (2009) Comparison of near infrared laser excitation wavelengths and its influence on the interrogation of seized drugs-of-abuse by Raman spectroscopy. J Raman Spectrosc 40(12): 1974-1983.

60. Yang W, Wu H, Qian J, Chandler L, Lieber C, et al. (2012) Multi-wavelength excitation Raman spectrometers and microscopes for measurements of real-world samples. Proc SPIE 8546: 854603.

61. Vitek P, Ali EMA, Edwards HGM, Jehlicka J, Cox R, et al. (2012) Evaluation of portable Raman spectrometer with $1064 \mathrm{~nm}$ excitation for geological and forensic applications. Spectrochim Acta A 86: 320-327.

62. Noonan KY, Tonge LA, Fenton OS, Damiano DB, Frederick KA (2009) Rapid classification of simulated street drug mixtures using Raman spectroscopy and principal component analysis. Appl Spectrosc 63(7): 742-747.

63. Ng PHR, Walker S, Tahtouh M, Reedy B (2009) Detection of illicit substances in fingerprints by infrared spectral imaging. Anal Bioanal Chem 394(8): 2039-2048. 